\title{
A BGG-TYPE RESOLUTION FOR TENSOR MODULES OVER GENERAL LINEAR SUPERALGEBRA
}

\author{
SHUN-JEN CHENG ${ }^{\dagger}$, JAE-HOON KWON ${ }^{\dagger \dagger}$, AND NGAU LAM ${ }^{\dagger \dagger}$
}

\begin{abstract}
We construct a Bernstein-Gelfand-Gelfand type resolution in terms of direct sums of Kac modules for the finite-dimensional irreducible tensor representations of the general linear superalgebra. As a consequence it follows that the unique maximal submodule of a corresponding reducible Kac module is generated by its proper singular vector.
\end{abstract}

Key words: Bernstein-Gelfand-Gelfand resolution, singular vector, Kac module, general linear superalgebra.

Mathematics Subject Classifications (2000): 17B67.

\section{INTRODUCTION}

The classical result of Bernstein-Gelfand-Gelfand [BGG] resolves a finite-dimensional irreducible module over a finite-dimensional semi-simple Lie algebra in terms of direct sums of Verma modules. Such a resolution is sometimes called a strong BGG resolution. In $[\mathrm{L}, \mathrm{RC}]$ it was shown that the finite-dimensional simple modules may also be resolved in terms of direct sums of generalized Verma modules.

While BGG resolutions have been known to exist for integrable representations over Kac-Moody algebras (see e.g. [RCW, K]), virtually nothing is known even for finitedimensional simple Lie superalgebras. However, what seems to be known to experts is that, in general, the finite-dimensional simple modules over a finite-dimensional simple Lie superalgebra cannot be resolved in terms of Verma modules. For example, even the natural representation of the Lie superalgebra $\mathfrak{s l}(1 \mid 2)$ (or $\mathfrak{g l}(1 \mid 2)$ ) cannot have a resolution in terms of Verma modules (see Example 5.1).

It is therefore surprising that resolutions for a large class of finite-dimensional representations of the general linear superalgebra $\mathfrak{g l}(m \mid n)$ in terms of Kac modules do exist. The purpose of this article is to construct such a resolution for every irreducible tensor module (see Section 2.3) of $\mathfrak{g l}(m \mid n)$.

Roughly the idea of the construction is to exploit the connection between the irreducible tensor representations of the Lie superalgebra $\mathfrak{g l}(m \mid n)$ and the polynomial representations of the general linear algebra $\mathfrak{g l}(m+n)$ in the limit $n \rightarrow \infty$. This allows us to construct a "weak" resolution. The strong resolution is then obtained from the weak version using Brundan's Kazhdan-Lusztig theory of $\mathfrak{g l}(m \mid n)[\mathrm{B}]$.

\footnotetext{
${ }^{\dagger}$ Partially supported by an NSC-grant of the ROC and an Academia Sinica Investigator grant.

${ }^{\dagger \dagger}$ Partially supported by KRF-grant 2005-070-C00004.

${ }^{\dagger \dagger}$ Partially supported by an NSC-grant 96-2115-M-006-008-MY3 of the ROC.
} 
All vector spaces, algebras and tensor products are over the complex number field $\mathbb{C}$.

\section{Preliminaries}

Let $m \in \mathbb{N}$ and $n \in \mathbb{N} \cup\{\infty\}$, and set $I(m \mid n)=\{-m, \cdots,-1,1, \ldots, n\}$ for $n \in \mathbb{N}$, and $I(m \mid n)=\{-m, \cdots,-1\} \cup \mathbb{N}$ for $n=\infty$. Let $\mathcal{P}_{m \mid n}$ denote the set of partitions $\lambda=\left(\lambda_{-m}, \cdots, \lambda_{-1}, \lambda_{1}, \lambda_{2}, \cdots\right)$ with $\lambda_{1} \leq n$. The set $\mathcal{P}_{m \mid \infty}$ is the set of all partitions. For a partition $\lambda$, we use $\lambda^{\prime}, \ell(\lambda)$, and $|\lambda|$ to denote its conjugate, length, and size, respectively.

2.1. The Lie algebra $\mathfrak{g l}(m+n)$. We let $\mathbb{C}^{m+n}$ stand for the complex space of dimension $m+n$ with the standard basis $\left\{e_{i} \mid i \in I(m \mid n)\right\}$. Let $\mathfrak{g}=\mathfrak{g l}(m+n)$ be the general linear algebra which acts naturally on $\mathbb{C}^{m+n}$. In the case of $n=\infty$, we let $\mathfrak{g}$ consist of linear transformations vanishing on all but finitely many $e_{j}$ 's. Denote by $\left\{E_{i j} \mid i, j \in I(m \mid n)\right\}$ the set of elementary matrices in $\mathfrak{g}$. Then $\left\{E_{j j} \mid j \in I(m \mid n)\right\}$ spans the standard Cartan subalgebra $\mathfrak{h}=\mathfrak{h}_{n}$, while $\left\{E_{i j} \mid i \leq j\right\}$ spans the standard Borel subalgebra. For $\lambda \in \mathfrak{h}^{*}$ we denote by $L(\mathfrak{g}, \lambda)$ the irreducible highest weight $\mathfrak{g}$-module with highest weight $\lambda$.

Let $\epsilon_{j} \in \mathfrak{h}^{*}$ be determined by $\left\langle\epsilon_{j}, E_{i i}\right\rangle=\delta_{i j}$ for $i, j \in I(m \mid n)$. Let $\alpha_{i}=\epsilon_{i}-\epsilon_{i+1}$, for $i \in I(m \mid n)$ such that $i+1 \in I(m \mid n)$, and $\alpha_{-1}=\epsilon_{-1}-\epsilon_{1}$. Then the set $\left\{\alpha_{i}\right\}$ is a set of simple roots of $\mathfrak{g}^{\prime}=[\mathfrak{g}, \mathfrak{g}]$, and we denote the set of positive and negative roots by $\Delta^{ \pm}$, respectively. Let $\Delta_{0}^{ \pm}=\Delta^{ \pm} \cap\left(\sum_{i \neq-1} \mathbb{Z} \alpha_{i}\right)$ and $\Delta^{ \pm}(0)=\Delta^{ \pm} \backslash \Delta_{0}^{ \pm}$.

Let $\left\{\alpha_{i}^{\vee}\right\}$ denote the corresponding simple coroots and let $\left\{e_{i}, f_{i}, \alpha_{i}^{\vee}\right\}$ be the corresponding Chevalley generators of $\mathfrak{g}^{\prime}$. Let $\rho_{c} \in \mathfrak{h}^{*}$ be determined by $\left\langle\rho_{c}, E_{j j}\right\rangle=-j$ for $j<0$, and $\left\langle\rho_{c}, E_{j j}\right\rangle=1-j$ for $j>0$.

The Lie algebra $\mathfrak{g}$ has a $\mathbb{Z}$-gradation determined by the eigenvalues of the operator $\frac{1}{2}\left(\sum_{i<0} E_{i i}-\sum_{j>0} E_{j j}\right)$. We have

$$
\mathfrak{g}=\mathfrak{g}_{-1} \oplus \mathfrak{g}_{0} \oplus \mathfrak{g}_{+1} .
$$

Note that $\mathfrak{g}_{0} \cong \mathfrak{g l}(m) \oplus \mathfrak{g l}(n)$ and $\mathfrak{g}_{-1} \cong \mathbb{C}^{m *} \otimes \mathbb{C}^{n}$ as $\mathfrak{g}_{0}$-modules. Set $\mathfrak{p}:=\mathfrak{g}_{0} \oplus \mathfrak{g}_{+1}$ and let $L^{0}(\lambda)$ be the irreducible representation of $\mathfrak{g}_{0}$ with highest weight $\lambda \in \mathfrak{h}^{*}$. We extend $L^{0}(\lambda)$ trivially to a $\mathfrak{p}$-module, for which we also write $L^{0}(\lambda)$. Denote the generalized Verma module by

$$
V(\mathfrak{g}, \lambda):=\operatorname{Ind}_{\mathfrak{p}}^{\mathfrak{g}} L^{0}(\lambda)
$$

2.2. The Lie superalgebra $\mathfrak{g l}(m \mid n)$. Now we let $\mathbb{C}^{m \mid n}$ stand for the complex superspace of dimension $(m \mid n)$ with the standard basis $\left\{\bar{e}_{i} \mid i \in I(m \mid n)\right\}$. We assume that $\operatorname{deg} \bar{e}_{i}=0$ and 1 if $i<0$ and $i>0$, respectively. Let $\overline{\mathfrak{g}}=\mathfrak{g l}(m \mid n)$ be the general linear superalgebra acting naturally on $\mathbb{C}^{m \mid n}$. For $n=\infty$, we use a similar convention as before. Denote by $\left\{\bar{E}_{i j} \mid i, j \in I(m \mid n)\right\}$ the set of elementary matrices in $\overline{\mathfrak{g}}$. Then $\left\{\bar{E}_{j j} \mid j \in I(m \mid n)\right\}$ spans the standard Cartan subalgebra $\overline{\mathfrak{h}}=\overline{\mathfrak{h}}_{n}$, while $\left\{\bar{E}_{i j} \mid i \leq j\right\}$ spans the standard Borel subalgebra $\overline{\mathfrak{b}}$. For $\lambda \in \overline{\mathfrak{h}}^{*}$, we denote by $L(\overline{\mathfrak{g}}, \lambda)$ the irreducible highest weight $\overline{\mathfrak{g}}$-module with highest weight $\lambda$. 
Let $\delta_{j} \in \overline{\mathfrak{h}}^{*}$ be determined by $\left\langle\delta_{j}, \bar{E}_{i i}\right\rangle=\delta_{i j}$ and let $\rho_{s} \in \overline{\mathfrak{h}}^{*}$ be determined by $\left\langle\rho_{s}, \bar{E}_{j j}\right\rangle=-j$ for $i, j \in I(m \mid n)$. Let $\beta_{i}=\delta_{i}-\delta_{i+1}$ for $i \in I(m \mid n)$ such that $i+1 \in$ $I(m \mid n)$, and $\beta_{-1}=\delta_{-1}-\delta_{1}$. Then $\left\{\beta_{i}\right\}$ is a set of simple roots of $\overline{\mathfrak{g}}^{\prime}=[\overline{\mathfrak{g}}, \overline{\mathfrak{g}}]$.

The Lie superalgebra $\overline{\mathfrak{g}}$ also has a $\mathbb{Z}$-gradation determined by the eigenvalues of the of the operator $\frac{1}{2}\left(\sum_{i<0} \bar{E}_{i i}-\sum_{j>0} \bar{E}_{j j}\right)$. We have

$$
\overline{\mathfrak{g}}=\overline{\mathfrak{g}}_{-1} \oplus \overline{\mathfrak{g}}_{0} \oplus \overline{\mathfrak{g}}_{+1} .
$$

Note that $\overline{\mathfrak{g}}_{0} \cong \mathfrak{g}_{0}$ and $\overline{\mathfrak{g}}_{-1} \cong \mathbb{C}^{m *} \otimes \mathbb{C}^{n}$ as $\overline{\mathfrak{g}}_{0}$-modules. We set $\overline{\mathfrak{p}}:=\overline{\mathfrak{g}}_{0} \oplus \overline{\mathfrak{g}}_{+1}$. Given $\lambda \in \overline{\mathfrak{h}}^{*}$, we may extend $L^{0}(\lambda)$ trivially to a $\overline{\mathfrak{p}}$-module, which we also denote by $L^{0}(\lambda)$. Define the Kac module to be

$$
V(\overline{\mathfrak{g}}, \lambda):=\operatorname{Ind} \frac{\overline{\mathfrak{g}}}{\overline{\mathfrak{p}}} L^{0}(\lambda) .
$$

Definition 1. A $\overline{\mathfrak{g}}$-module $V$ is said to have a Kac flag if it has a filtration of $\overline{\mathfrak{g}}$-modules of the form

$$
0=V_{0} \subseteq V_{1} \subseteq V_{2} \subseteq \cdots \subseteq V_{l-1} \subseteq V_{l}=V
$$

such that $V_{j} / V_{j-1}$ is isomorphic to a Kac module for $j=1, \ldots, l$.

Definition 2. Let $n \in \mathbb{N} \cup\{\infty\}$. Given a sequence of integers of the form

$$
\mu=\left(\mu_{-m}, \cdots, \mu_{-1}, \mu_{1}, \mu_{2}, \cdots\right),
$$

with $\mu_{k}=0$ for $k \gg 0$ when $n=\infty$, and

$$
\mu=\left(\mu_{-m}, \cdots, \mu_{-1}, \mu_{1}, \mu_{2}, \cdots, \mu_{n}\right)
$$

when $n \in \mathbb{N}$, we may interpret it as $\sum_{i \geq-m, i \neq 0} \lambda_{i} \epsilon_{i} \in \mathfrak{h}_{n}^{*}$ or $\sum_{i \geq-m, i \neq 0} \lambda_{i} \delta_{i} \in \overline{\mathfrak{h}}_{n}^{*}$. Suppose now that $\mu$ as in $(2.1)$ such that $\left(\mu_{1}, \mu_{2}, \cdots\right)$ is a partition. We define $\mu^{\natural}$ to be the integer sequence

$$
\mu^{\natural}:=\left(\mu_{-m}, \cdots, \mu_{-1}, \mu_{1}^{\prime}, \mu_{2}^{\prime}, \cdots\right) .
$$

Let $\widetilde{x}_{m \mid n}$ be the set of integer sequences of the form (2.1) with $\mu_{j} \geq \mu_{j+1}$, for all $j<n$ with $j \neq 0,-1$. Let $x_{m \mid n} \subseteq \widetilde{X}_{m \mid n}$ consist of those $\mu$ 's such that $\left(\mu_{1}, \mu_{2}, \cdots\right)$ is a partition. For $\mu \in X_{m \mid n}, \mu^{\natural}$ is well-defined, and the map $\mu \rightarrow \mu^{\natural}$ is a bijection on $X_{m \mid \infty}$.

2.3. Irreducible tensor $\mathfrak{g l}(m \mid n)$-modules. The tensor powers of $\mathbb{C}^{m \mid n}$ are completely reducible as $\overline{\mathfrak{g}}$-modules. Indeed the irreducible representations that appear in these decompositions are as follows. An irreducible representation of $\overline{\mathfrak{g}}$ appears as a component of $\left(\mathbb{C}^{m \mid n}\right)^{\otimes k}$ if and only if it is of the form $L\left(\overline{\mathfrak{g}}, \lambda^{\natural}\right)$, where $\lambda \in \mathcal{P}_{m \mid n}$ with $|\lambda|=k[\mathrm{~S}, \mathrm{BR}]$. We call these irreducible $\overline{\mathfrak{g}}$-modules irreducible tensor $\overline{\mathfrak{g}}$-modules.

Let $\lambda \in \mathcal{P}_{m \mid \infty}$. Clearly as $\mathfrak{g}_{0}$-modules $L(\mathfrak{g}, \lambda)$ and $L\left(\overline{\mathfrak{g}}, \lambda^{\natural}\right)$ are direct sums of $L^{0}(\eta)$ with $\eta \in X_{m \mid \infty}$. We have the following description of irreducible tensor $\overline{\mathfrak{g}}$-modules.

Proposition 2.1. Assume that $n=\infty$. For $\lambda \in \mathcal{P}_{m \mid \infty}$ and $\eta \in X_{m \mid \infty}$, the $\mathfrak{g}_{0}$-module $L^{0}(\eta)$ is an irreducible component of $L(\mathfrak{g}, \lambda)$ if and only if the $\overline{\mathfrak{g}}_{0}$-module $L^{0}\left(\eta^{\natural}\right)$ is an irreducible component of $L\left(\overline{\mathfrak{g}}, \lambda^{\natural}\right)$. Furthermore, their multiplicities coincide. 
Proof. This is an immediate consequence of the well-known fact that the character of $L\left(\overline{\mathfrak{g}}, \lambda^{\natural}\right)$ is given by the so-called Hook Schur function associated with $\lambda^{\natural}$ [BR, Theorem $6.10]$.

Remark 2.1. For a partition $\lambda$ with $\ell(\lambda) \leq m+n$ and $k \geq 0$, it is easy to see that $\Lambda^{k}\left(\mathfrak{g}_{-1}\right) \otimes L(\mathfrak{g}, \lambda)$ as a $\mathfrak{g}_{0}$-module decomposes into a direct sum of irreducible $\mathfrak{g}_{0}$-modules with highest weights belonging to $X_{m \mid n}$. Similarly, for $\lambda \in \mathcal{P}_{m \mid n}$ and $k \geq 0, \Lambda^{k}\left(\overline{\mathfrak{g}}_{-1}\right) \otimes$ $L\left(\overline{\mathfrak{g}}, \lambda^{\natural}\right)$ as a $\overline{\mathfrak{g}}_{0}$-module decomposes into a direct sum of irreducible $\overline{\mathfrak{g}}_{0}$-modules of the form $L^{0}(\mu)$ with $\mu \in X_{m \mid n}$.

\section{Eigenvalues of Casimir operators}

Throughout this section, we assume that $n=\infty$ unless otherwise specified.

We fix a symmetric bilinear form $(\cdot \mid \cdot)_{c}$ on $\mathfrak{h}^{*}$ satisfying

$$
\left(\lambda \mid \epsilon_{i}\right)_{c}=\left\langle\lambda, E_{i i}\right\rangle, \quad \lambda \in \mathfrak{h}^{*}, i \in I(m \mid n) .
$$

By defining $\left(\alpha_{i}^{\vee} \mid \alpha_{j}^{\vee}\right)_{c}:=\left(\alpha_{i} \mid \alpha_{j}\right)_{c}$ for simple coroots $\alpha_{i}^{\vee}$ and $\alpha_{j}^{\vee}$, we obtain a symmetric bilinear form on the Cartan subalgebra of $\mathfrak{g}^{\prime}$, which can be extended to a non-degenerate invariant symmetric bilinear form on $\mathfrak{g}^{\prime}$ such that

$$
\left(e_{i} \mid f_{j}\right)_{c}=\delta_{i j} .
$$

Since every root space $\mathfrak{g}_{\alpha}$ is one-dimensional, we can choose a basis $\left\{u_{\alpha}\right\}$ of $\mathfrak{g}_{\alpha}$ for $\alpha \in \Delta^{+}$and a dual basis $\left\{u^{\alpha}\right\}$ of $\mathfrak{g}_{-\alpha}$ with respect to $(\cdot \mid \cdot)_{c}$.

Let $V=\bigoplus_{\mu} V_{\mu}$ be a highest weight $\mathfrak{g}$-module, where $V_{\mu}$ denotes the $\mu$-weight space of $V$. Define $\Gamma_{1}: V \rightarrow V$ to be the linear map that acts as the scalar $\left(\mu+2 \rho_{c} \mid \mu\right)_{c}$ on $V_{\mu}$. Let $\Gamma_{2}:=2 \sum_{\alpha \in \Delta^{+}} u^{\alpha} u_{\alpha}$. The Casimir operator (cf. [J]) is defined to be

$$
\Omega:=\Gamma_{1}+\Gamma_{2} .
$$

It follows from (3.1) and (3.2) that $\Omega$ commutes with the action of $\mathfrak{g}$ on $V$ (cf. [J, Proposition 3.6]). Thus, if $V$ is generated by a highest weight vector with highest weight $\lambda$, then $\Omega$ acts on $V$ as the scalar $\left(\lambda+2 \rho_{c} \mid \lambda\right)_{c}$.

To produce the Casimir operator for $\overline{\mathfrak{g}}$ we fix a symmetric bilinear form $(\cdot \mid \cdot)_{s}$ on $\overline{\mathfrak{h}}^{*}$ satisfying

$$
\left(\lambda \mid \delta_{i}\right)_{s}=-\operatorname{sign}(i)\left\langle\lambda, E_{i i}\right\rangle, \quad \lambda \in \overline{\mathfrak{h}}^{*}, i \in I(m \mid n) .
$$

An analogous argument allows us to generalize the construction above and define the Casimir operator $\bar{\Omega}$ of the Lie superalgebra $\overline{\mathfrak{g}}$ that acts on a highest weight module with highest weight $\gamma \in \overline{\mathfrak{h}}^{*}$ as the scalar $\left(\gamma+2 \rho_{s} \mid \gamma\right)_{s}$. We omit the details.

We will need the Weyl group of $\mathfrak{g l}(m+\infty)$ in the sequel. For each $\alpha_{j}$, define simple reflection $\sigma_{j}$ by

$$
\sigma_{j}(\mu):=\mu-\left\langle\mu, \alpha_{j}^{\vee}\right\rangle \alpha_{j},
$$

where $\mu \in \mathfrak{h}^{*}$. Let $W$ be the subgroup of $\operatorname{Aut}\left(\mathfrak{h}^{*}\right)$ generated by the $\sigma_{j}$ 's. For each $w \in W$, we let $l(w)$ denote the length of $w$. We have an action on $\mathfrak{h}$ given by $\sigma_{j}(h)=$ $h-\left\langle\alpha_{j}, h\right\rangle \alpha_{j}^{\vee}$ for $h \in \mathfrak{h}$, so that $\langle w(\mu), w(h)\rangle=\langle\mu, h\rangle$ for $\mu \in \mathfrak{h}^{*}$ and $h \in \mathfrak{h}$. We also define

$$
w \circ \mu:=w\left(\mu+\rho_{c}\right)-\rho_{c}, \quad w \in W, \mu \in \mathfrak{h}^{*} .
$$


Consider $W_{0}$ the subgroup of $W$ generated by $\sigma_{j}$ with $j \neq-1$. Let

$$
W^{0}:=\left\{w \in W \mid w\left(\Delta^{-}\right) \cap \Delta^{+} \subseteq \Delta^{+}(0)\right\} .
$$

It is well-known that $W=W_{0} W^{0}$ and $W^{0}$ is the set of the minimal length representatives of the right coset space $W_{0} \backslash W$ (cf. [K, 1.3.17]). For $k \in \mathbb{Z}_{+}$, set

$$
W_{k}^{0}:=\left\{w \in W^{0} \mid l(w)=k\right\} .
$$

Given $\lambda \in \mathcal{P}_{m \mid \infty}$, we have $\left\langle\lambda, \alpha_{j}^{\vee}\right\rangle \in \mathbb{Z}_{+}$for all $j$. Since $w \in W^{0}$ implies that $w^{-1}\left(\Delta_{0}^{+}\right) \subseteq \Delta^{+}$, we obtain $\left\langle w \circ \lambda, \alpha_{j}^{\vee}\right\rangle \in \mathbb{Z}_{+}$, for all $j \neq-1$, and $w \circ \lambda \in X_{m \mid \infty}$.

The following proposition is well-known from the theory of standard modules over generalized Kac-Moody algebras (see e.g. [J, Proposition 3.11]).

Proposition 3.1. For $\lambda \in \mathcal{P}_{m \mid \infty}$ and $\eta \in \mathcal{X}_{m \mid \infty}$, the irreducible $\mathfrak{g}_{0}$-module $L^{0}(\eta)$ is a component of $\Lambda^{k}\left(\mathfrak{g}_{-1}\right) \otimes L(\mathfrak{g}, \lambda)$ with $\left(\eta+2 \rho_{c} \mid \eta\right)_{c}=\left(\lambda+2 \rho_{c} \mid \lambda\right)_{c}$ if and only if there exists $w \in W_{k}^{0}$ with $w \circ \lambda=\eta$. Furthermore each such $L^{0}(\eta)$ appears with multiplicity one.

Lemma 3.1. For $\lambda \in \mathcal{P}_{m \mid \infty}$ and $\eta \in X_{m \mid \infty}, L^{0}(\eta)$ is an irreducible $\mathfrak{g}_{0}$-module in $\Lambda^{k}\left(\mathfrak{g}_{-1}\right) \otimes L(\mathfrak{g}, \lambda)$ if and only if $L^{0}\left(\eta^{\natural}\right)$ is an irreducible $\overline{\mathfrak{g}}_{0}$-module in $\Lambda^{k}\left(\overline{\mathfrak{g}}_{-1}\right) \otimes L\left(\overline{\mathfrak{g}}, \lambda^{\natural}\right)$. Furthermore, the multiplicities are the same.

Proof. The symmetric [H, Theorem 2.1.2] and skew-symmetric [H, Theorem 4.1.4] $(\mathfrak{g l}, \mathfrak{g l})$-Howe dualities give the precise decompositions of $\Lambda^{k}\left(\overline{\mathfrak{g}}_{-1}\right) \cong S^{k}\left(\mathbb{C}^{m *} \otimes \mathbb{C}^{n}\right)$ and $\Lambda^{k}\left(\mathfrak{g}_{-1}\right) \cong \Lambda^{k}\left(\mathbb{C}^{m *} \otimes \mathbb{C}^{n}\right)$ as $\mathfrak{g}_{0}$-modules, respectively. From these decompositions one sees that $L^{0}(\eta)$ is an irreducible component in $\Lambda^{k}\left(\mathfrak{g}_{-1}\right)$ if and only if $L^{0}\left(\eta^{\natural}\right)$ is an irreducible component in $\Lambda^{k}\left(\overline{\mathfrak{g}}_{-1}\right)$. This fact combined with Proposition 2.1 and the compatibility of $\downarrow$ under tensor products completes the proof.

We need the following combinatorial lemma.

Lemma 3.2. Let $\lambda=\left(\lambda_{1}, \lambda_{2}, \cdots, \lambda_{N}\right)$ be a partition with $\ell(\lambda) \leq N$. For $1 \leq i \leq N$ the sets $\left\{\lambda_{i}^{\prime}-i+\frac{1}{2} \mid \lambda_{i}^{\prime}-i+\frac{1}{2}>0\right\}$ and $\left\{-\lambda_{i}+i-\frac{1}{2} \mid \lambda_{i}-i+\frac{1}{2}<0\right\}$ are disjoint. Moreover, $\left\{\lambda_{i}^{\prime}-i+\frac{1}{2} \mid \lambda_{i}^{\prime}-i+\frac{1}{2}>0\right\} \cup\left\{-\lambda_{i}+i-\frac{1}{2} \mid \lambda_{i}-i+\frac{1}{2}<0\right\}$ is a permutation of the set $\left\{\frac{1}{2}, \frac{3}{2}, \cdots, N-\frac{1}{2}\right\}$.

Proof. The sets $\left\{\lambda_{i}^{\prime}-i+\frac{1}{2} \mid \lambda_{i}^{\prime}-i+\frac{1}{2}>0\right\},\left\{-\lambda_{i}+i-\frac{1}{2} \mid \lambda_{i}-i+\frac{1}{2}<0\right\},\left\{\frac{1}{2}, \frac{3}{2}, \cdots, N-\frac{1}{2}\right\}$ are denoted by $A, B$ and $C$, respectively. We first observe that the sequence $\left\{\lambda_{i}^{\prime}-i+\right.$ $\left.\frac{1}{2}\right\}_{i=1}^{N}$ is strictly decreasing, while $\left\{-\lambda_{i}+i-\frac{1}{2}\right\}_{i=1}^{N}$ is strictly increasing. Also $A$ and $B$ are subsets of $C$. Since $\lambda_{i}^{\prime}-i+\frac{1}{2}>0$ if and only if $\lambda_{i}-i+\frac{1}{2}>0$, we have $i<j$ for all $\lambda_{i}^{\prime}-i+\frac{1}{2} \in A$ and $-\lambda_{j}+j-\frac{1}{2} \in B$. Furthermore, the sum of the cardinality of $A$ and the cardinality of $B$ equals the cardinality of $C$. So it is enough to show $A \cap B=\emptyset$. Suppose that $\lambda_{i}^{\prime}-i+\frac{1}{2} \in A$ and $-\lambda_{j}+j-\frac{1}{2} \in B$ with $\lambda_{i}^{\prime}-i+\frac{1}{2}=-\lambda_{j}+j-\frac{1}{2}$. We have $i<j$ and $\lambda_{i}^{\prime}+\lambda_{j}=i+j-1$. If $\lambda_{i}^{\prime} \geq j$, we have $\lambda_{i}^{\prime}+\lambda_{j} \geq j+i>j+i-1$. If $\lambda_{i}^{\prime}<j$, we have $\lambda_{i}^{\prime}+\lambda_{j}<j+(i-1)=j+i-1$. In either case, $\lambda_{i}^{\prime}+\lambda_{j} \neq i+j-1$. Thus we have $A \cap B=\emptyset$, which completes the proof.

Lemma 3.3. For $\mu \in X_{m \mid \infty}$, we have $\left(\mu+2 \rho_{c} \mid \mu\right)_{c}=\left(\mu^{\natural}+2 \rho_{s} \mid \mu^{\natural}\right)_{s}$. 
Proof. A direct calculation shows that the lemma is equivalent to the following identity for a partition $\mu=\left(\mu_{1}, \mu_{2}, \cdots\right)$ :

$$
\sum_{j>0} \mu_{j}^{2}-\sum_{j>0} 2(j-1) \mu_{j}=\sum_{j>0} 2 j \mu_{j}^{\prime}-\sum_{j>0}\left(\mu_{j}^{\prime}\right)^{2} .
$$

This identity is equivalent to

$$
\sum_{j=1}^{N}\left[\left(\mu_{j}-\left(j-\frac{1}{2}\right)\right)^{2}+\left(\mu_{j}^{\prime}-\left(j-\frac{1}{2}\right)\right)^{2}\right]=2 \sum_{j=1}^{N}\left(j-\frac{1}{2}\right)^{2},
$$

where $N \geq \max \left(\ell(\mu), \ell\left(\mu^{\prime}\right)\right)$. However (3.3) follows readily from Lemma 3.2 applied to the partitions $\mu$ and $\mu^{\prime}$.

Proposition 3.2. For $\lambda \in \mathcal{P}_{m \mid \infty}$ and $\mu \in \overline{\mathfrak{h}}^{*}$, the irreducible $\overline{\mathfrak{g}}_{0}$-module $L^{0}(\mu)$ is a component of $\Lambda^{k}\left(\overline{\mathfrak{g}}_{-1}\right) \otimes L\left(\overline{\mathfrak{g}}, \lambda^{\natural}\right)$ with $\left(\mu+2 \rho_{s} \mid \mu\right)_{s}=\left(\lambda^{\natural}+2 \rho_{s} \mid \lambda^{\natural}\right)_{s}$ if and only if there exists $w \in W_{k}^{0}$ with $\mu=(w \circ \lambda)^{\natural}$. Furthermore, each such $L^{0}(\mu)$ appears with multiplicity one.

Proof. Let $L^{0}(\mu)$ be an irreducible $\overline{\mathfrak{g}}_{0}$-module in $\Lambda^{k}\left(\overline{\mathfrak{g}}_{-1}\right) \otimes L\left(\overline{\mathfrak{g}}, \lambda^{\natural}\right)$. By Remark 2.1, we have $\mu=\eta^{\natural}$ for some $\eta \in X_{m \mid \infty}$. By Lemma 3.1, $L^{0}(\eta)$ is an irreducible component of $\Lambda^{k}\left(\mathfrak{g}_{-1}\right) \otimes L(\mathfrak{g}, \lambda)$ with the same multiplicity. By Lemma 3.3, if $\left(\mu+2 \rho_{s} \mid \mu\right)_{s}=$ $\left(\lambda^{\natural}+2 \rho_{s} \mid \lambda^{\natural}\right)_{s}$, then we have $\left(\eta+2 \rho_{c} \mid \eta\right)_{c}=\left(\lambda+2 \rho_{c} \mid \lambda\right)_{c}$. Furthermore by Proposition 3.1, $\eta=w \circ \lambda$ for some $w \in W_{k}^{0}$, and the multiplicity of $L^{0}(\mu)$ is one.

Conversely, if $\mu=(w \circ \lambda)^{\natural}$ for some $w \in W_{k}^{0}$, then by Lemma 3.3 we get

$$
\left(\mu+2 \rho_{s} \mid \mu\right)_{s}=\left(\lambda^{\natural}+2 \rho_{s} \mid \lambda^{\natural}\right)_{s} .
$$

By Proposition 3.1, $L^{0}(w \circ \lambda)$ appears in $\Lambda^{k}\left(\mathfrak{g}_{-1}\right) \otimes L(\mathfrak{g}, \lambda)$ with multiplicity one. Hence by Lemma $3.1 L^{0}(\mu)$ also appears in $\Lambda^{k}\left(\overline{\mathfrak{g}}_{-1}\right) \otimes L\left(\overline{\mathfrak{g}}, \lambda^{\natural}\right)$ with multiplicity one.

\section{WEAK BGG-TyPE RESOLUtions FOR IRREDUCIBLE TENSOR $\mathfrak{g l}(m \mid n)$-MODULES}

Since $\overline{\mathfrak{g}} / \overline{\mathfrak{p}}$ is a $\overline{\mathfrak{p}}$-module, $D_{k}:=U(\overline{\mathfrak{g}}) \otimes_{U(\overline{\mathfrak{p}})} \Lambda^{k}(\overline{\mathfrak{g}} / \overline{\mathfrak{p}})$ is a $\overline{\mathfrak{g}}$-module with $\overline{\mathfrak{g}}$ acting on the first factor, for $k \geq 0$. Define the sequence

$$
\cdots \stackrel{\partial_{k+1}}{\longrightarrow} D_{k} \stackrel{\partial_{k}}{\longrightarrow} D_{k-1} \stackrel{\partial_{k-1}}{\longrightarrow} \cdots \stackrel{\partial_{1}}{\longrightarrow} D_{0} \stackrel{\epsilon}{\longrightarrow} \mathbb{C} \longrightarrow 0,
$$

where $\epsilon$ is the augmentation map from $U(\overline{\mathfrak{g}})$ to $\mathbb{C}$ and

$$
\partial_{k}\left(a \otimes \bar{x}_{1} \bar{x}_{2} \cdots \bar{x}_{k}\right):=\sum_{j=1}^{k} a x_{j} \otimes \bar{x}_{1} \cdots \widehat{x}_{j} \cdots \bar{x}_{k},
$$

for $a \in U(\overline{\mathfrak{g}})$ and $x_{i} \in \overline{\mathfrak{g}}$. Here $\bar{x}_{j}$ denotes the image of $x_{j}$ in $\overline{\mathfrak{g}} / \overline{\mathfrak{p}}$ under the natural map. One easily checks that the $\partial_{k}$ 's are well-defined $U(\overline{\mathfrak{g}})$-maps and (4.1) is a chain complex. The exactness of (4.1) follows, for example, from the exactness of the dual of the Koszul complex [K, Appendix D.13] (see also [KK]).

For $\lambda \in \mathcal{P}_{m \mid n}$ and $k \geq 0, Y_{k}:=D_{k} \otimes L\left(\overline{\mathfrak{g}}, \lambda^{\natural}\right)$ is a $\overline{\mathfrak{g}}$-module. Tensoring (4.1) with $L\left(\overline{\mathfrak{g}}, \lambda^{\natural}\right)$ we obtain an exact sequence [GL, K, J]

$$
\cdots \stackrel{d_{k+1}}{\longrightarrow} Y_{k} \stackrel{d_{k}}{\longrightarrow} Y_{k-1} \stackrel{d_{k-1}}{\longrightarrow} \cdots \stackrel{d_{1}}{\longrightarrow} Y_{0} \stackrel{d_{0}}{\longrightarrow} L\left(\overline{\mathfrak{g}}, \lambda^{\natural}\right) \longrightarrow 0,
$$


where $d_{k}:=\partial_{k} \otimes 1$ for $k>0$ and $d_{0}:=\epsilon \otimes 1$.

Let $V$ be a $\overline{\mathfrak{g}}$-module, on which the action of $\overline{\mathfrak{g}}_{+1}$ is locally nilpotent. We define

$$
V^{c}:=\left\{v \in V \mid(\bar{\Omega}-c)^{l} v=0 \text { for } l \gg 0\right\},
$$

i.e. $V^{c}$ is the generalized $\bar{\Omega}$-eigenspace corresponding to the eigenvalue $c \in \mathbb{C}$. Clearly we have $V=\bigoplus_{c \in \mathbb{C}} V^{c}$. Put

$$
c_{\lambda}=\left(\lambda^{\natural}+2 \rho_{s} \mid \lambda^{\natural}\right)_{s} .
$$

The restriction of (4.2) to the generalized $c_{\lambda}$-eigenspace of $\bar{\Omega}$ produces a resolution of $\overline{\mathfrak{g}}$-modules

$$
\cdots \stackrel{d_{k+1}}{\longrightarrow} Y_{k}^{c_{\lambda}} \stackrel{d_{k}}{\longrightarrow} Y_{k-1}^{c_{\lambda}} \stackrel{d_{k-1}}{\longrightarrow} \cdots \stackrel{d_{1}}{\longrightarrow} Y_{0}^{c_{\lambda}} \stackrel{d_{0}}{\longrightarrow} L\left(\overline{\mathfrak{g}}, \lambda^{\natural}\right) \longrightarrow 0 .
$$

Proposition 4.1. Assume that $n=\infty$. For $\lambda \in \mathcal{P}_{m \mid \infty}$, we have a resolution of $\overline{\mathfrak{g}}-$ modules of the form

$$
\cdots \stackrel{d_{k+1}}{\longrightarrow} Z_{k} \stackrel{d_{k}}{\longrightarrow} Z_{k-1} \stackrel{d_{k-1}}{\longrightarrow} \cdots \stackrel{d_{1}}{\longrightarrow} Z_{0} \stackrel{d_{0}}{\longrightarrow} L\left(\overline{\mathfrak{g}}, \lambda^{\natural}\right) \longrightarrow 0
$$

such that each $Z_{k}$ has a Kac flag. Furthermore, $Z_{k} \cong \bigoplus_{w \in W_{k}^{0}} V\left(\overline{\mathfrak{g}},(w \circ \lambda)^{\natural}\right)$ as $\overline{\mathfrak{g}}_{-1}+\overline{\mathfrak{g}}_{0^{-}}$ modules.

Proof. Observe that $Y_{k} \cong U(\overline{\mathfrak{g}}) \otimes_{U(\overline{\mathfrak{p}})}\left(\Lambda^{k}(\overline{\mathfrak{g}} / \overline{\mathfrak{p}}) \otimes L\left(\overline{\mathfrak{g}}, \lambda^{\natural}\right)\right)$. Suppose that as $\overline{\mathfrak{g}}_{0}$-module, we have $\Lambda^{k}(\overline{\mathfrak{g}} / \overline{\mathfrak{p}}) \otimes L\left(\overline{\mathfrak{g}}, \lambda^{\natural}\right) \cong \bigoplus_{\mu \in \mathcal{J}} L^{0}(\mu)$ for some multiset of weights $\mathcal{J}$. The $\overline{\mathfrak{p}}$ module $\Lambda^{k}(\overline{\mathfrak{g}} / \overline{\mathfrak{p}}) \otimes L\left(\overline{\mathfrak{g}}, \lambda^{\natural}\right)$ has a composition series, where the multiset of composition factors is precisely the multiset of $\overline{\mathfrak{p}}$-module $L^{0}(\mu), \mu \in \mathcal{J}$. Thus $Y_{k}$ has a Kac flag and $Y_{k} \cong \bigoplus_{\mu \in \mathcal{J}} V(\overline{\mathfrak{g}}, \mu)$ as $\overline{\mathfrak{g}}_{-1}+\overline{\mathfrak{g}}_{0}$-modules. Now $\bar{\Omega}$ acts on $V(\overline{\mathfrak{g}}, \mu)$ as the scalar $\left(\mu+2 \rho_{s} \mid \mu\right)_{s}$, and hence $Z_{k}=Y_{k}^{c_{\lambda}} \cong \bigoplus_{\mu} V(\overline{\mathfrak{g}}, \mu)$, where the summation is over all $\mu \in \mathcal{J}$ such that $\left(\mu+2 \rho_{s} \mid \mu\right)_{s}=\left(\lambda^{\natural}+2 \rho_{s} \mid \lambda^{\natural}\right)_{s}$. Proposition 3.2 now says that this set is precisely $\left\{(w \circ \lambda)^{\natural} \mid w \in W_{k}^{0}\right\}$.

Corollary 4.1. Assume that $n \in \mathbb{N}$. For $\lambda \in \mathcal{P}_{m \mid n}$, we have a resolution of $\overline{\mathfrak{g}}$-modules of the form

$$
\cdots \stackrel{d_{k+1}}{\longrightarrow} Z_{k, n} \stackrel{d_{k}}{\longrightarrow} Z_{k-1, n} \stackrel{d_{k-1}}{\longrightarrow} \cdots \stackrel{d_{1}}{\longrightarrow} Z_{0, n} \stackrel{d_{0}}{\longrightarrow} L\left(\overline{\mathfrak{g}}, \lambda^{\natural}\right) \longrightarrow 0
$$

such that each $Z_{k, n}$ has a Kac flag. Furthermore, $Z_{k, n} \cong \bigoplus_{w \in W_{k}^{0}} V\left(\overline{\mathfrak{g}},(w \circ \lambda)^{\natural}\right)$ as $\overline{\mathfrak{g}}_{-1}+\overline{\mathfrak{g}}_{0}$-modules. Here, by definition we have $V\left(\overline{\mathfrak{g}}, \nu^{\natural}\right)=0$ for $\nu \in X_{m \mid \infty}$ with $\nu_{1}>n$.

Proof. The corollary follows from applying the truncation functor $\mathfrak{t r}_{n}$ [CWZ, Definition 4.4] upon the resolution in Proposition 4.1 and using the facts that the truncation functor is an exact functor and is compatible with both irreducible and Kac modules [CWZ, Corollary 4.6].

\section{Strong BGG-type Resolutions for irreducible tensor $\mathfrak{g l}(m \mid n)$-MOdules}

For $n \in \mathbb{N}$ recall the definition of the super Bruhat ordering for $\overline{\mathfrak{g}}=\mathfrak{g l}(m \mid n)$ on $\widetilde{X}_{m \mid n}$ in $[\mathrm{B}, \S 2-\mathrm{b}]$, which we denote by $\preccurlyeq$. This gives a partial ordering on $\widetilde{x}_{m \mid n}$. We can restrict $\preccurlyeq$ to $X_{m \mid n}$, which can be defined for $X_{m \mid \infty}$ as well (cf. [CWZ, Section 2.3]). Now we may also regard $\widetilde{x}_{m \mid n}$ as weights of $\mathfrak{g}=\mathfrak{g l}(m+n)$. In doing so the usual Bruhat 
ordering of $\mathfrak{g}$ determines a partial ordering $\leq$ on $\widetilde{X}_{m \mid n}$ (see e.g. [CWZ, Section 2.2]), which restricts to $X_{m \mid n}$, and which in turn can be defined for $X_{m \mid \infty}$ as well. We have the following.

Lemma 5.1. [CWZ, Lemma 6.6] Let $\lambda, \mu \in X_{m \mid \infty}$. Then $\lambda \preccurlyeq \mu$ if and only if $\lambda^{\natural} \leq \mu^{\natural}$.

In the remainder of this section we assume that $n \in \mathbb{N}$ unless otherwise specified.

Lemma 5.2. Let $n \in \mathbb{N}$ and $\lambda, \mu \in \widetilde{x}_{m \mid n}$. Suppose that $\mu \npreceq \lambda$. Then

$$
\operatorname{Hom}_{\overline{\mathfrak{g}}}(V(\overline{\mathfrak{g}}, \mu), V(\overline{\mathfrak{g}}, \lambda))=0 .
$$

Proof. Suppose that $\operatorname{Hom}_{\overline{\mathfrak{g}}}(V(\overline{\mathfrak{g}}, \mu), V(\overline{\mathfrak{g}}, \lambda)) \neq 0$. Then $L(\overline{\mathfrak{g}}, \mu)$ is a composition factor of the Kac module $V(\overline{\mathfrak{g}}, \lambda)$. It follows from [B, Corollary 3.36 (i)] and [B, Theorem 4.37] that $\mu \preccurlyeq \lambda$.

Lemma 5.3. Let $n \in \mathbb{N}$ and $\mu \in \widetilde{X}_{m \mid n}$. Suppose that $M$ is a finite-dimensional $\overline{\mathfrak{g}}$ module with a Kac flag

$$
0=M_{0} \subseteq M_{1} \subseteq M_{2} \subseteq \cdots \subseteq M_{l}=M
$$

and $\operatorname{Hom}_{\overline{\mathfrak{g}}}\left(M_{i} / M_{i-1}, V(\overline{\mathfrak{g}}, \mu)\right)=0$ for all $i=1, \cdots, l$. Then

$$
\operatorname{Hom}_{\overline{\mathfrak{g}}}(M, V(\overline{\mathfrak{g}}, \mu))=0 .
$$

Proof. Since $M$ is finite-dimensional we have $M_{i} / M_{i-1} \cong V\left(\overline{\mathfrak{g}}, \mu_{i}\right)$ with $\mu_{i} \in \widetilde{X}_{m \mid n}$ for all $i$. Consider the exact sequence

$$
0 \rightarrow M_{1} \rightarrow M \rightarrow M / M_{1} \rightarrow 0 .
$$

Noting that $M / M_{1}$ has a Kac flag of length $l-1$, the lemma follows easily from the long exact sequence and induction on $l$.

Lemma 5.4. Let $n \in \mathbb{N}$ and $\lambda, \mu \in X_{m \mid n}$. Suppose that $\lambda$ and $\mu$ are not comparable under the super Bruhat ordering. Then

$$
\operatorname{Ext}^{1}(V(\overline{\mathfrak{g}}, \lambda), V(\overline{\mathfrak{g}}, \mu))=0 .
$$

Proof. Consider $P(\lambda)$ the projective cover (in the category of finite-dimensional $\overline{\mathfrak{g}}-$ modules) of $L(\overline{\mathfrak{g}}, \lambda)$. We have an exact sequence

$$
0 \rightarrow K \rightarrow P(\lambda) \rightarrow V(\overline{\mathfrak{g}}, \lambda) \rightarrow 0 .
$$

Now $P(\lambda)$ has a Kac flag [Z, Proposition 2.5] and hence so has $K$. By [B, Theorem 4.37], $P(\lambda)$ is a tilting module and if $V(\overline{\mathfrak{g}}, \gamma)$ with $\gamma \neq \lambda$ appears in a Kac flag of $P(\lambda)$, then $\gamma \in \widetilde{X}_{m \mid n}$ and $\gamma \succ \lambda$.

Now the induced long exact sequence from (5.1) gives rise to the following exact sequence

$$
\operatorname{Hom}_{\overline{\mathfrak{g}}}(K, V(\overline{\mathfrak{g}}, \mu)) \rightarrow \operatorname{Ext}^{1}(V(\overline{\mathfrak{g}}, \lambda), V(\overline{\mathfrak{g}}, \mu)) \rightarrow 0 .
$$

Since all $V(\overline{\mathfrak{g}}, \gamma)$ that appears in the Kac flag of $K$ are such that $\gamma \succ \lambda$, we see that $\gamma \npreceq \mu$ by hypothesis. Thus by Lemmas 5.2 and $5.3, \operatorname{Hom}_{\overline{\mathfrak{g}}}(K, V(\overline{\mathfrak{g}}, \mu))=0$, and the lemma follows. 
Theorem 5.1. For $n \in \mathbb{N}$ and $\lambda \in \mathcal{P}_{m \mid n}$, we have a resolution of $\overline{\mathfrak{g}}$-modules of the form

$$
\cdots \stackrel{d_{k+1}}{\longrightarrow} Z_{k, n} \stackrel{d_{k}}{\longrightarrow} Z_{k-1, n} \stackrel{d_{k-1}}{\longrightarrow} \cdots \stackrel{d_{1}}{\longrightarrow} Z_{0, n} \stackrel{d_{0}}{\longrightarrow} L\left(\overline{\mathfrak{g}}, \lambda^{\natural}\right) \rightarrow 0,
$$

where $Z_{k, n} \cong \bigoplus_{w \in W_{k}^{0}} V\left(\overline{\mathfrak{g}},(w \circ \lambda)^{\natural}\right)$ as $\overline{\mathfrak{g}}$-modules. As before, by definition, we have $V\left(\overline{\mathfrak{g}}, \nu^{\natural}\right)=0$ for $\nu \in \mathcal{X}_{m \mid \infty}$ with $\nu_{1}>n$.

Proof. We have a natural embedding of $x_{m \mid N} \stackrel{\iota_{N, N+1}}{\longrightarrow} X_{m \mid N+1}$ for any $N \in \mathbb{N}$. Also we have the truncation map $x_{m \mid N+1} \stackrel{\operatorname{Tr}_{N+1, N}}{\longrightarrow} x_{m \mid N}$ [CWZ, Section 6.6] that sends an element $\lambda=\left(\lambda_{-m}, \cdots, \lambda_{N+1}\right)$ to $\lambda=\left(\lambda_{-m}, \cdots, \lambda_{N}\right)$, if $\lambda_{N+1}=0$, and to $\emptyset$, otherwise. The usual Bruhat orderings of $\mathcal{X}_{m \mid N}$ and $\mathcal{X}_{m \mid N+1}$ are compatible in the following sense:

(i) For $\lambda, \mu \in X_{m \mid N}$, one has $\lambda \leq \mu$ if and only if $\iota_{N, N+1}(\lambda) \leq \iota_{N, N+1}(\mu)$.

(ii) For $\lambda, \mu \in X_{m \mid N+1}$ with $\operatorname{Tr}_{N+1, N}(\lambda) \neq \emptyset, \operatorname{Tr}_{N+1, N}(\mu) \neq \emptyset$, one has $\lambda \leq \mu$ if and only if $\operatorname{Tr}_{N+1, N}(\lambda) \leq \operatorname{Tr}_{N+1, N}(\mu)$.

Thus the Bruhat ordering of $X_{m \mid N}$ is compatible with that of $X_{m \mid \infty}$.

We view $\lambda$ as a weight of $\mathfrak{g l}(m+\infty)$ and so as an element in $X_{m \mid \infty}$. For a fixed $j \in \mathbb{N}$, it is not hard to see that the weights $\left\{w \circ \lambda \mid w \in W_{j}^{0}\right\}$ form a finite set and they all may be regarded as lying in the same $x_{m \mid N}$, for $N \gg 0$. Thus we may regard them all as weights of $\mathfrak{g l}(m+N)$ for some $N \gg 0$. But for such weights, it is well-known from classical theory of semi-simple Lie algebras that they are not comparable under the usual Bruhat ordering (see e.g. [K, Lemma 1.3.16]). Thus viewing them as weights of $\mathfrak{g l}(m+\infty)$, they are not comparable under the Bruhat ordering, either. Hence, by Lemma 5.1, the weights $(w \circ \lambda)^{\natural}$ are not comparable under the super Bruhat ordering of $\mathfrak{g l}(m \mid \infty)$. The theorem now follows from a similar compatibility of the super Bruhat orderings of $\mathfrak{g l}(m \mid \infty)$ and of $\mathfrak{g l}(m \mid n)$, Lemma 5.4 and Corollary 4.1.

Remark 5.1. Note that $W$ above is the infinite Weyl group of $\mathfrak{g l}(m+\infty)$, even though we are considering the finite-dimensional Lie superalgebra $\mathfrak{g l}(m \mid n)$.

Remark 5.2. Theorem 5.1 has the counterpart in the case of $n=\infty$ as well.

Recall that for $\lambda, \mu \in \widetilde{X}_{m \mid n}$ with $\lambda \succcurlyeq \mu$ there is a relative length function defined in $[\mathrm{B}, \S 3-\mathrm{g}]$, which we denote by $\bar{\ell}(\mu, \lambda)$. Fix $\lambda \in \mathcal{P}_{m \mid n}$ so that $\lambda^{\natural} \in \widetilde{X}_{m \mid n}$. For $\mu \in \widetilde{X}_{m \mid n}$ with $\lambda^{\natural} \succcurlyeq \mu$ define an absolute length function by

$$
\bar{\ell}(\mu):=\bar{\ell}\left(\mu, \lambda^{\natural}\right) .
$$

We can now formulate Theorem 5.1 intrinsically without referring to the infinite Weyl group of $\mathfrak{g l}(m+\infty)$ as follows.

Theorem 5.2. For $n \in \mathbb{N}$ and $\lambda \in \mathcal{P}_{m \mid n}$, we have a resolution of $\overline{\mathfrak{g}}$-modules of the form

$$
\cdots \stackrel{d_{k+1}}{\longrightarrow} Z_{k, n} \stackrel{d_{k}}{\longrightarrow} Z_{k-1, n} \stackrel{d_{k-1}}{\longrightarrow} \cdots \stackrel{d_{1}}{\longrightarrow} Z_{0, n} \stackrel{d_{0}}{\longrightarrow} L\left(\overline{\mathfrak{g}}, \lambda^{\natural}\right) \rightarrow 0,
$$

where $Z_{k, n} \cong \bigoplus_{\bar{\ell}(\mu)=k} V(\overline{\mathfrak{g}}, \mu)$ as $\overline{\mathfrak{g}}$-modules. 
Proof. For $\nu, \mu \in \widetilde{X}_{m \mid n}$ recall Brundan's Kazhdan-Lusztig polynomials $l_{\mu \nu}(q)$ of [B, (2.18)]. By [B, Theorem 4.51] and [Z, Theorem 5.1] we have the following cohomological interpretation:

$$
l_{\mu \nu}\left(-q^{-1}\right)=\sum_{i=0}^{\infty} \operatorname{dim}\left[\operatorname{Hom}_{\overline{\mathfrak{g}}_{0}}\left(L^{0}(\mu), \mathrm{H}^{i}\left(\overline{\mathfrak{g}}_{+1} ; L(\overline{\mathfrak{g}}, \nu)\right)\right)\right] q^{i} .
$$

The calculation of the $\overline{\mathfrak{g}}_{+1}$-cohomology groups in [CZ, Corollary 4.14] now implies that

$$
l_{\mu \lambda^{\natural}}\left(-q^{-1}\right)= \begin{cases}q^{k}, & \text { if there exists } w \in W_{k}^{0} \text { with } \mu=(w \circ \lambda)^{\natural} \text { and }(w \circ \lambda)_{1} \leq n, \\ 0, & \text { otherwise. }\end{cases}
$$

From [B, Corollary 3.45] we conclude that for such $\mu$ we have $k=\bar{\ell}(\mu)$. On the other hand if $\mu \in \widetilde{X}_{m \mid n}$ with $\bar{\ell}(\mu)=k$, then [B, Corollary 3.45] implies that $l_{\mu \lambda^{\natural}}\left(-q^{-1}\right) \neq 0$ and hence $\mu$ is of the form $(w \circ \lambda)^{\natural}$ with $w \in W_{k}^{0}$. Thus for $\mu \in \widetilde{X}_{m \mid n}$ the condition that there exists $w \in W_{k}^{0}$ with $\mu=(w \circ \lambda)^{\natural}$ is equivalent to the condition that $\bar{\ell}(\mu)=k$. This together with Theorem 5.1 completes the proof.

We record the following corollary of the proof of Theorem 5.2.

Corollary 5.1. Let $\lambda \in \mathcal{P}_{m \mid n}$. As a $\overline{\mathfrak{g}}_{0}$-module we have, for all $k \in \mathbb{Z}_{+}$,

$$
\mathrm{H}^{k}\left(\overline{\mathfrak{g}}_{+1} ; L\left(\overline{\mathfrak{g}}, \lambda^{\natural}\right)\right) \cong \bigoplus_{\bar{\ell}(\mu)=k} L^{0}(\mu) .
$$

We conclude with an example, which shows that finite-dimensional irreducible representations over a simple Lie superalgebra cannot be resolved in terms of direct sums Verma modules in general.

Example 5.1. Let $\lambda \in \overline{\mathfrak{h}}^{*}$ and let $\mathbb{C}_{\lambda}$ denote the one-dimensional $\overline{\mathfrak{h}}$-module that transforms by $\lambda$. We extend $\mathbb{C}_{\lambda}$ trivially to a $\overline{\mathfrak{b}}$-module and denote by $M(\overline{\mathfrak{g}}, \lambda)=\operatorname{Ind} \frac{\overline{\mathfrak{g}}}{\mathfrak{b}} \mathbb{C}_{\lambda}$ the Verma module of highest weight $\lambda$. Suppose that $L(\overline{\mathfrak{g}}, \lambda)$ can be resolved in terms of Verma modules. Then we have an exact sequence of $\overline{\mathfrak{g}}$-modules of the form

$$
\cdots \longrightarrow \bigoplus_{i \in I} M\left(\overline{\mathfrak{g}}, \mu_{i}\right) \stackrel{\psi}{\longrightarrow} M(\overline{\mathfrak{g}}, \lambda) \stackrel{\phi}{\longrightarrow} L(\overline{\mathfrak{g}}, \lambda) \longrightarrow 0
$$

and $\operatorname{Hom}_{\overline{\mathfrak{g}}}\left(M\left(\overline{\mathfrak{g}}, \mu_{i}\right), M(\overline{\mathfrak{g}}, \lambda)\right) \neq 0$, for all $i \in I$. It follows that there exist singular vectors $v_{i}$ of weight $\mu_{i}$ in $M(\overline{\mathfrak{g}}, \lambda) . \operatorname{Im} \psi=\operatorname{Ker} \phi$ implies that the unique maximal submodule of $M(\overline{\mathfrak{g}}, \lambda)$ must be generated by the proper singular vectors of $M(\overline{\mathfrak{g}}, \lambda)$.

Now consider $\lambda=\delta_{-1}$ and $\overline{\mathfrak{g}}=\mathfrak{g l}(1 \mid 2)$ or $\overline{\mathfrak{g}}=\mathfrak{s l}(1 \mid 2)$. In the sequel we will suppress $\overline{\mathfrak{g}}$. One can show by a direct calculation that the only proper singular vectors in the Verma module $M\left(\delta_{-1}\right)$ are scalar multiples of either $\bar{E}_{2,1} v$ or $\bar{E}_{1,-1} \bar{E}_{2,1} v$, where $v$ is a highest weight vector of $M\left(\delta_{-1}\right)$. If $M_{1}$ is the submodule of $M\left(\delta_{-1}\right)$ generated by $\bar{E}_{2,1} v$, then $M_{1}$ is the submodule generated by all proper singular vectors of $M\left(\delta_{-1}\right)$. But $\operatorname{dim}\left(M\left(\delta_{-1}\right) / M_{1}\right)=4$ by the PBW Theorem and, since $\operatorname{dim} L\left(\delta_{-1}\right)=3$, it follows that $M_{1}$ cannot be the unique maximal submodule of $M\left(\delta_{-1}\right)$. Thus $L\left(\delta_{-1}\right)$ cannot have a resolution in terms of Verma modules. We note that $M\left(\delta_{-1}\right) / M_{1}$ is isomorphic to the Kac module of highest weight $\delta_{-1}$ and $\bar{E}_{1,-1} \bar{E}_{2,-1} v$ is a singular vector in $M\left(\delta_{-1}\right) / M_{1}$. 
Theorem 5.1, the fact that $\mathrm{H}^{1}\left(\overline{\mathfrak{g}}_{+1} ; L\left(\overline{\mathfrak{g}}, \lambda^{\natural}\right)\right)$ is irreducible, and the discussion in Example 5.1 imply the following.

Corollary 5.2. Let $n \in \mathbb{N}$ and $\lambda \in \mathcal{P}_{m \mid n}$. The unique maximal submodule of a reducible $V\left(\overline{\mathfrak{g}}, \lambda^{\natural}\right)$ is generated by the proper singular vector of $V\left(\overline{\mathfrak{g}}, \lambda^{\natural}\right)$.

\section{REFERENCES}

[BGG] I. Bernstein, I. Gelfand and S. Gelfand: Differential operators on the base affine space and a study of $\mathfrak{g}$-modules. Lie groups and their representations (Proc. Summer School, Bolyai Janos Math. Soc., Budapest, 1971), pp. 21-64. Halsted, New York, 1975.

[B] J. Brundan: Kazhdan-Lusztig Polynomials and Character Formulae for the Lie Superalgebra $\mathfrak{g l}(m \mid n)$, J. Amer. Math. Soc. 16 (2003) 185-231.

[BR] A. Berele and A. Regev: Hook Young diagrams with applications to combinatorics and representations of Lie superalgebras, Adv. Math. 64 (1987) 118-175.

[CWZ] S.-J. Cheng, W. Wang and R. B. Zhang: Super Duality and Kazhdan-Lusztig Polynomials, Trans. Amer. Math. Soc., to appear, math.RT/0409016.

[CZ] S.-J. Cheng and R. B. Zhang: Analogue of Kostant's u-cohomology formula for the general linear superalgebra, Int. Math. Res. Not. 2004 (2004) 31-53.

[GL] H. Garland and J. Lepowsky: Lie Algebra Homology and the Macdonald-Kac Formulas, Invent. Math. 34 (1976) 37-76.

[H] R. Howe: Remarks on classical invariant theory, Trans. Amer. Math. Soc. 313 (1989), 539570. Perspectives on Invariant Theory: Schur Duality, Multiplicity-free Actions and Beyond, The Schur Lectures, Israel Math. Conf. Proc. 8, Tel Aviv (1992) 1-182.

[J] E. Jurisich: An Exposition of Generalized Kac-Moody Algebras. Lie algebras and their representations (Seoul, 1995) 121-159, Contemp. Math. 194, Amer. Math. Soc., Providence, RI, 1996.

[K] S. Kumar: Kac-Moody groups, their flag varieties and representation theory. Progress in Mathematics, 204. Birkhauser Boston, Inc., Boston, MA, 2002.

[KK] S. J. Kang and J. H. Kwon: Graded Lie superalgebras, supertrace formula, and orbit Lie superalgebra, Proc. Lond. Math. Soc. 81 (2000), 675-724.

[L] J. Lepowsky: A generalization of the Bernstein-Gelfand-Gelfand resolution, J. Algebra 49 (1977) 496-511.

[RC] A. Rocha-Caridi: Splitting Criteria for $\mathfrak{g}$-Modules Induced from a Parabolic and the Bernstein-Gelfand-Gelfand Resolution of a Finite Dimensional Irreducible $\mathfrak{g}$-Module, Trans. Amer. Math. Soc. 262 (1980) 335-366.

[RCW] A. Rocha-Caridi and N. Wallach: Projective modules over graded Lie algebras I, Math. Z. 180 (1982) 151-177.

[S] A. Sergeev: The Tensor Algebra of the Identity Representation as a Module over the Lie Superalgebras $g l(n, m)$ and $Q(n)$, Math. USSR Sbornik 51 (1985) 419-427.

[Z] Y. M. Zou: Categories of finite-dimensional weight modules over type I classical Lie superalgebras. J. Algebra 180 (1996) 459-482.

Institute of Mathematics, Academia Sinica, Taipei, Taiwan 11529

E-mail address: chengsj@math.sinica.edu.tw

Department of Mathematics, University of Seoul, 90, Cheonnong-Dong, DongdaemunGU, Seoul 130-743, Korea

E-mail address: jhkwon@uos.ac.kr

Department of Mathematics, National Cheng-Kung University, Tainan, Taiwan 70101

E-mail address: nlam@mail.ncku.edu.tw 